\title{
Gut Microbial succession during conventionalization of germfree chicken.
}

Milton Thomas ${ }^{1,2}$, Supapit Wongkuna ${ }^{1,2,3}$, Sudeep Ghimire ${ }^{1,2}$, Kinchel C. Doerner ${ }^{4}$, Aaron

Singery $^{1,2}$, Eric Nelson ${ }^{1,2}$, Tofuko Woyengo ${ }^{5}$, Surang Chankhamhaengdecha ${ }^{6}$, Tavan Janvilisri ${ }^{4}$, and Joy Scaria ${ }^{1,2} *$

${ }^{1}$ Department of Veterinary and Biomedical Sciences, South Dakota State University, Brookings,

SD, USA.

${ }^{2}$ South Dakota Center for Biologics Research and Commercialization, SD, USA.

${ }^{3}$ Department of Biochemistry, Faculty of Science, Mahidol University, Bangkok, Thailand

${ }^{4}$ Department of Biology and Microbiology, South Dakota State University, Brookings, SD, USA.

${ }^{5}$ Department of Animal Science, South Dakota State University, Brookings, SD, USA.

${ }^{6}$ Department of Biology, Faculty of Science, Mahidol University, Bangkok, Thailand

*Address correspondence to: Joy Scaria

Email: joy.scaria@sdstate.edu 


\begin{abstract}
A gnotobiotic chicken model was developed to study the succession of intestinal microflora from hatching to 18 days of age. Intestinal samples were collected from a local population of feral chickens and administered orally to germ-free 3 day old chicks. Animals were enthanized on 0, 9 and 18 days of age and intestinal samples were collected and subjected to genomic analysis. The five most prevalent phyla were Bacteroidetes (45.73 $\pm 3.35 \%)$, Firmicutes $(36.47 \pm 2.60 \%)$, Proteobacteria (8.28 $\pm 0.91 \%)$, Actinobacteria (5.09 $\pm 0.52 \%)$, and Spriochetes $(2.10 \pm 0.38 \%)$. Principle coordinate analysis indicated the 0,9 day and 18 day variables clustered together and the microbial communities changed temporally. The Morista-Horn index values ranged from 0.72 to 1 , indicating the communities at 0,9 or 18 days were more similar than dissimilar. The predicted functional profiles of the microbiomes of 0,9 and 18 days were also similar. These results indicate the gnotobiotic chicks stably maintain the phylogentic diversity and predicted metabolic functionality of the inoculum community.
\end{abstract}

\title{
Importance
}

The domestic chicken is the cornerstone of animal agriculture worldwide with a flock population exceeding 40 billion birds/year. It serves as the economically valuable source of protein globally. Microbiome of poultry has important effects on chicken growth, feed conversion, immune status and pathogen resistance. The significance of our research is in developing a gnotobiotic chicken model to study chicken gut microbiota function. Our experimental model shows that young germfree chicks are able to colonize diverse set of gut bacteria. Therefore, besides using this model to study mechanisms of gut microbiota interactions in the chicken gut, our model could be also used for applied aspects such as determining the safety and efficacy of new probiotic strains derived from chicken gut microbiota. 


\section{Key words}

Microbiota, Succession, Salmonella, Germfree, Gnotobiotic, Feral Chicken, competitive exclusion, metagenome 


\section{Introduction}

The chicken gut microbiota influences nutrient utilization $(1,2)$, immune development (3), endocrine activity (4), development of gastrointestinal tract (5), and detoxification, thus contributing to the improved performance of the birds. The chicken gastrointestinal tract harbors complex communities of bacteria $(6,7)$. However, cecum harbors the most number of commensals and contains up to $10^{11} / \mathrm{g}$ organisms (8) and therefore was widely studied. In addition to commensal bacteria, cecum also could harbor enteric pathogens that pose both avian and zoonotic health risk (9). The commensals could prevent the colonization of pathogens by competitive exclusion (10) and through the production of bacteriocins $(11,12)$.

Several experiments were conducted previously to study the microbial succession in broiler chicken intestinal tract $(7,13-15)$. Also, studies were performed to determine the effect of gut microbes on feed utilization and conversion $(1,16)$. However, these experiments used 16s RNA sequencing or culture-based techniques. The $16 \mathrm{~S}$ rRNA sequencing is inherently limited due to bias introduced during PCR reactions. Also, the data has lower resolution and is less efficient in predicting the functional properties of the microbiome. The accuracy of culture-based enumeration of the bacterial population is negatively affected by the inability to grow all the bacteria in culture conditions. In these respect, shotgun metagenomics provides a comprehensive representation of both taxonomical and functional properties of the microbiome. The two studies that used shotgun metagenomics for analyzing chicken microbiome were limited by the number of birds used in those experiments $(6,17)$.

Feral chickens are derived from domestic chickens that are released to wild and survive many generations. Living in the wilderness induces differences in the feeding habits and social behavioral patterns. Previous research in wild fowls and turkeys have shown that the microbial 
communities in these birds vary considerably from the domesticated counterparts (18-21).

Similar to these findings, we hypothesized that adult feral microbiome could be substantially diverse from the microbial population of the commercial poultry. Use of feral chicken microbiome as probiotic in commercial poultry practices could increase the microbial diversity and thereby possibly provide colonization resistance against enteric pathogens.

The objective of this experiment was to analyze the microbial succession in gnotobiotic chickens when inoculated with adult feral chicken microbiome using shotgun metagenomics. Our findings suggested that feral chicken microbiome could colonize successfully in the young chicken gut without causing detrimental health effects to the host.

\section{Results}

\section{Presence of Salmonella in the Feral Chicken Gut and Sterility of the Isolator}

The donor material was collected anaerobically from the cecum and colon contents of 6 feral chickens and immediately was screened for the presence of Salmonella by streaking on XLT4 agar plates. All plates were negative for Salmonella growth after $24 \mathrm{~h}$ incubation at $37^{0} \mathrm{C}$ indicating Salmonella was not present in the gut of the feral chickens. The sterility of the isolator and chicks were examined by culturing fecal droppings and swabs from gnotobiotic isolators and found to be negative for bacterial colonies indicating the isolator and chicks were germ-free.

\section{Phylogenetic distribution of microbiome in gnotobiotic chicken gut compared to inoculum}

\section{Microbiome composition in the inoculum and cecal contents at phylum level:}

A taxonomical abundance table with phyla-level distribution was generated in MG-RAST using RefSeq database. The five major phyla in all the samples were Bacteroidetes, Firmicutes, Proteobacteria, Actinobacteria, and Spirochetes (Figure 1). The proportion of Bacteroidetes in 
the feral chicken inoculum was $66.42 \%$ but was lower in $9 \mathrm{~d}(47.49 \pm 4.38 \%)$ and $18 \mathrm{~d}$ samples $(45.73 \pm 3.35 \%)$. There was no significant difference between the $9 \mathrm{~d}$ and $18 \mathrm{~d}$ samples for Bacteroidetes. The abundance of Firmicutes in the inoculum was $18 \%$ and increased to $27.24 \pm$ $3.88 \%$ in $9 \mathrm{~d}$ and to $36.47 \pm 2.60 \%$ in $18 \mathrm{~d}$ samples. The Firmicutes abundance in the chicken gut significantly increased by $18 \mathrm{~d}$ compared to $9 \mathrm{~d}(\mathrm{P}>0.05)$. The $9 \mathrm{~d}$ samples showed a major shift in the proportion of Proteobacteria which nearly doubled compared to inoculum $(9.65 \%$ in inoculum vs $16.64 \pm 2.78$ in $9 \mathrm{~d}$ chicken). However, the Proteobacteria level decreased to that in the inoculum by $18 \mathrm{~d}(8.28 \pm 0.91 \%)$ and was statistically lower than $9 \mathrm{~d}$ samples $(\mathrm{P}>0.05)$. This is in concurrence with the previous findings that facultative anaerobes proliferate initially in the infant gut followed by the outgrowth of the obligate anaerobic bacterial community due to oxygen depletion in the gut (22). Similar to Firmicutes, the abundance of Actinobacteria also increased temporally. In the inoculum, the fraction of Actinobacteria was $1.62 \%$, whereas, in $9 \mathrm{~d}$ and $18 \mathrm{~d}$ samples, the abundance increased to $3.99 \pm 1.41$ and $5.09 \pm 0.52 \%$ respectively. The percentages of Spirochetes remained similar in the inoculum and gnotobiotic chicken samples at $9 \mathrm{~d}$ and $18 \mathrm{~d}(1.52,2.58 \pm 0.94$, and $2.10 \pm 0.38 \%)$.

\section{Microbiome composition in the inoculum and cecal contents at genera level:}

At the genus level, the inoculum, $9 \mathrm{~d}$, and $18 \mathrm{~d}$ samples was composed predominantly of Bacteroides (Figure 2). However, the abundance was higher in the inoculum $(52.85 \%)$ compared to $9 \mathrm{~d}$ (mean $\pm \mathrm{SEM} ; 37.87 \pm 3.09 \%)$ and $18 \mathrm{~d}(35.52 \pm 2.53 \%)$ samples. Clostridium increased in the $9 \mathrm{~d}(7.22 \pm 0.99 \%)$ and $18 \mathrm{~d}(9.72 \pm 0.74 \%)$ cecal contents compared to inoculum (5.16\%). The next abundant genus was Prevotella with the inoculum and gnotobiotic chicken samples indicating similar percentages. Escherichia, a member of the Proteobacteria, had higher abundance in the $9 \mathrm{~d}$ samples $(7.39 \pm 1.69 \%)$ compared to inoculum $(2.28 \%)$ and then 
decreased by $18 \mathrm{~d}(1.03 \pm 0.14 \%)$. Parabacteroides which represented $4.23 \%$ in the inoculum also decreased, similar to Bacteroides, to $2.26 \pm 0.25$ and $2.40 \pm 0.18 \%$ in $9 \mathrm{~d}$ and $18 \mathrm{~d}$ samples respectively. The other two major genera that increased in the cecal contents of gnotobiotic chicken compared to inoculum were Ruminococcus and Eubacterium which are members of Firmicute phylum.

\section{Principle coordinate analysis $(P C A)$ and $\beta$ diversity:}

The principal coordinate analysis (PCA) was calculated using euclidean distance as the similarity metric for clustering the metagenomes (Figure $3 \mathrm{~A}$ ). While the $9 \mathrm{~d}$ communities were randomly distributed across space, the $18 \mathrm{~d}$ communities were clustered together and separate from the control, which represented $70.6 \%$ of the variation and indicated the microbial communities evolved and matured temporally and attained a similar community profile. These findings are similar to the microbial succession occurring in a previously uninhabited environment such as infant gut where the microbial community attains maturity and stability in the initial years of life (23). Also, the community assemblage in the chicken gut by $18 \mathrm{~d}$ resembled closely with the inoculum when compared to $9 \mathrm{~d}$. Genera that were significantly altered in proportion between $9 \mathrm{~d}$ and $18 \mathrm{~d}$ samples are given in Figure $3 \mathrm{~B}$. Shigella and Escherichia each decreased by $18 \mathrm{~d}$ compared to $9 \mathrm{~d}$. Alternatively, the proportion of Selenomonas spp., Geobacillus spp., and Mitsuokella spp. increased by $18 \mathrm{~d}$. The read percentages of other bacteria that significantly increased by $18 \mathrm{~d}$ represented less than $0.1 \%$. Shotgun metagenomics was used to study the dynamics of the microbial community structure in the cecum of gnotobiotic chicken and the inoculum. The $\beta$-diversity represents the diversity in the compositional units between the samples. The values for Morisita-Horn index range from 0 to 1 where 1 indicates similar communities and 0 indicates dissimilar and are given 
in Figure 4A. All the values ranged between 0.72 and 1.0 indicating that the communities are more similar than dissimilar. However, individual variations in the colonization pattern were evident. For example, birds 4, 6 and 7 on $9 \mathrm{~d}$ and birds 3, 4, 6, and 7 on d 18 have dissimilar communities compared to the inoculum. However, the functional characteristics (Figure 4 B) of the communities were more similar between the samples. The range of Morisita-Horn index varied between 0.99 to 1 suggesting that the functional properties of inoculum and cecal samples at $9 \mathrm{~d}$ and $18 \mathrm{~d}$ from gnotobiotic birds were similar.

\section{Functional analysis of the cecal microbiome in the gnotobiotic chicken}

Analysis of functional categorization of the bacterial metagenome provides an understanding of the metabolic profile of the community. The metagenomes of feral chicken inoculum and cecal samples of gnotobiotic chicken on $9 \mathrm{~d}$ and $18 \mathrm{~d}$ were analyzed in MG-RAST pipeline using SEED subsystems database at level 2 hierarchy (Figure 5). The overall distribution pattern for ORFs that represented major cellular functions was similar for inoculum and gnotobiotic chicken microbiome on both $9 \mathrm{~d}$ and $18 \mathrm{~d}$. In all the metagenomes, the most abundant reads (approximately $22 \%$ ) represented genes that had an unknown function. Other predominant gene function belonged to categories such as protein biosynthesis, plant-prokaryote associations, RNA processing and modification, and central carbohydrate metabolism. These findings resembled the $\beta$ diversity for functional characteristics where all the communities exhibited similar profile.

\section{Discussion}

The major objective of this experiment was to develop a gnotobiotic model to investigate the microbial succession in the cecum of gnotobiotic chickens. Various methods of rearing gnotobiotic chicken have been described previously (15, 24-27). Gnotobiotic chickens have been 
reared using specially designed Gustafsson germ-free apparatus (25). However, more simplified methods were developed which made use of various disinfectants to reduce the bacterial load on eggs and sterile isolators $(15,24,26,27)$. Generally, the disinfectants used were mercuric chloride, quaternary ammonium, iodoform, sodium hypochlorite solutions and commercially available chlorine dioxide solutions. In this study, Sporicidin ${ }^{\circledR}$ was highly efficient in achieving disinfection without damaging the eggshells. Bacterial growth was not observed from samples collected from bird droppings and eggshells $2 \mathrm{~d}$ after hatching.

The conventionalization of gnotobiotic chickens using cecal microbial population derived from adult chickens has been previously conducted $(15,28)$. The major shortcoming of these studies was that the microbial community was identified using culture-based technique and only a few organisms could be identified. In this study, we used shotgun metagenomics to compare the microbiome of the donor material derived from apparently healthy feral chickens and the gnotobiotic chickens. By enriching for the microbial genomic DNA, shotgun metagenomics could be successfully performed using Miseq Illumina platform (29). This reduces the cost associated with using Hiseq which is the traditional platform used for shotgun metagenomics studies. Additionally, Miseq generates longer reads (250bp) compared to 150bp reads generated by Hiseq. The findings from this study indicated that gnotobiotic chicken model, when paired with next-generation sequencing techniques, could be an excellent tool to study the succession of gastrointestinal microbes in the chicken and could also be utilized in future experiments for studying the pathogenesis of enteric pathogens such as Salmonella.

The microbial population for inoculating gnotobiotic chickens in this study was collected from feral chickens that were Salmonella culture-negative. Feral chickens originated from domesticated birds that were released to the wild and have adapted to the wilderness through 
multiple generations. The process of feralization involves changes in social behavioral patterns, sexual selection, foraging requirements, and adaptation to predation in the wild. The expression of the genes that control these phenotypes also changes in the wild (30). Along with the host genetic changes, the microbiome could also diverge from the domesticated fowls. A study comparing the microbiome of wild and domesticated turkeys indicated that although the diversity and richness of the microbial population were similar, only $30 \%$ of the OTUs were shared between them (21). This suggested that feralization could induce adaptation of new bacterial genera to the host. Introduction of these new species to the domesticated poultry could possibly alter the microbial community in a beneficial way in the fight against enteric pathogens. In this study, we found that feral microbiome could successfully colonize in the young chicken gut without causing any detrimental effects to the health of the host.

There are few published reports on microbiome of feral chickens (Ferrario, Alessandri et al. 2017). However, the microbial population in the cecum of the pasture-housed chickens at the age of 2, 4, and 6 wk was studied recently using 16S sequencing (2). The birds were kept in conventional housing pens for first $2 \mathrm{wk}$ and were released to the pasture for up to $6 \mathrm{wk}$ of age. Firmicutes were the most abundant (59\%) at 2 wk of age which decreased to $47 \%$ by 6 wk of age. Contrarily, the relative abundance of Bacteroidetes increased from $31 \%$ at 2 wk of age to $41 \%$ after 4 wk of pasture-housing. A substantial presence of Proteobacteria (9\%) was also present in the cecal microbial population at 2 wk of age which decreased to $6 \%$ after pasturehousing. The most abundant genera in the 2 wk old chicken were Bacteroides, Ruminococaceae, and Lachnospiraceae. However, the proportion of all these genera decreased as the age progressed. Although the transient nature of microbiome with age is apparent from this study, the 
microbial profile may not be comparable to free-ranging feral chicken since all the birds in this experiment were fed on a formulated diet.

Conversely, the broiler chicken microbiome has been studied extensively using culturebased techniques $(8,10,28), 16 \mathrm{~S}$ sequencing $(13,14,31-34)$, and shotgun metagenomics $(6,17)$. The major difference in the microbial composition of feral chicken and broiler birds was Bacteroidetes predominated in the feral chicken microbiome $(66.4 \%)$. Firmicutes represented only $18.0 \%$ while Proteobacteria formed 9.7\%. Together, these 3 phyla constituted more than $94 \%$ of the microbiome in feral chicken. On the contrary, Firmicutes were found to be the most abundant phyla in the cecum of broiler chicken. In $5 \mathrm{wk}$ old broiler chickens, it was reported that $67 \%$ of the total sequences belonged to phylum Firmicutes (31). A similar finding was observed in the ceca of broiler chickens where Clostridiaceae-related sequences formed $65 \%$ of the $16 \mathrm{~S}$ rRNA clones derived from various age groups (14). Also, other studies that analyzed the effect of age on cecal microbial composition found that Firmicutes predominated and composed more than $90 \%$ in one-week-old chicken and decreased with age to $56 \%$ by $35 \mathrm{~d}(6,7,35)$. Among the Firmicutes, Ruminococcaceae and Lachnospiraceae were found to be the most abundant families $(7,33)$. An age-related lowering in the abundance of Ruminococcaceae and Lachnospiraceae and an increase in the abundance of the Clostridiaceae family in the cecal microbiome was also observed recently (7). Substantial presence of Proteobacteria was also reported in the previous experiments. In 3 day-old broiler chickens, approximately $15 \%$ of total $16 \mathrm{~S}$ rRNA sequences were identified as Proteobacteria (14), while in older chicken it formed $11-20 \%$ of the total microbiome $(31,33)$. Proteobacteria was reported as the most abundant phylum following Firmicutes in the cecum of broiler chickens in other studies $(6,7,35)$. Bacteroides was found in relatively less proportion or even absent in the cecum $(6,33)$. On the contrary, it was found that 
the proportion of Bacteroidetes increased from $2 \%$ at $15 \mathrm{~d}$ to $36 \%$ by $29 \mathrm{~d}$ of age (7). Similar to feral chicken microbiome, the presence of Actinobacteria was also detected in the cecum of broiler chicken in a recent study (35).

At the genus level, Bacteroides (52.9\%) were the most abundant organisms in the feral chicken microbiome. This was followed by Clostridium (5.2\%) and Prevotella (5.3\%), while Ruminococcus $(1.14 \%)$ and Lactobacillus $(0.44 \%)$ formed lower proportions. This is in contrary to the reports from broiler chicken cecal microbiome where Ruminococcus and Lactobacilli were found to be the predominant genera. In 3 day-old chicken cecum, Ruminococcus species formed $15.6 \%$ (14) of the total sequences and in 5 week-old, it was 6\% (31). Similarly, Lactobacillus species was detected at 7-8\% in broiler chicken cecum in these studies. A stable proportion of 16 $-23 \%$ Ruminococcus species in the total cecal microbiome which did not alter with age was observed by Ranjitkar et al. (7).

The gnotobiotic chicken microbiome from this study had different proportions of Bacteroidetes, Firmicutes, and Actinobacteria at both $9 \mathrm{~d}$ and $18 \mathrm{~d}$ compared to feral chicken inoculum. The abundance of Bacteroidetes was lower while Firmicutes and Actinobacteria were higher in conventionalized chicken compared to the feral chicken microbiome. Furthermore, in the conventionalized chickens used in this study, the proportion of phylum Bacteroidetes was relatively stable at $9 \mathrm{~d}$ and $18 \mathrm{~d}$ of age while the abundance of Firmicutes and Actinobacteria increased with age. The differences in feed and age of the birds could possibly explain these variations in the colonization profile. Dietary intervention is a primary driving force that causes alterations in the microbiome $(36,37)$. Feral chickens forage in the wild on a variety of feed which includes insects, berries, and worms while the gnotobiotic chicken was fed on poultry starter-diet. Another reason for the discrepancy between the feral and gnotobiotic chicken 
microbiome profiles could be that the inoculum was derived from pooled colon and cecal contents of feral chicken, while the analysis of gnotobiotic chicken microbiome was performed using the samples that were solely collected from the cecum.

Another finding was that although the proportion of Proteobacteria was higher at $9 \mathrm{~d}$ of age, it decreased by age and reached the level found in inoculum by $18 \mathrm{~d}$. At the genus level, there was a decrease in the abundance of Escherichia and Shigella, which are members of Proteobacteria. This shift is analogous to the microbial succession in infant gut where initial colonization is by Enterococcus and Escherichia, followed by Bifidobacterium and further by obligate anaerobes belonging to Firmicutes and Bacteroidetes (38-40). Similarly, a higher proportion of Escherichia was reported in young chicken which was later substituted by obligate anaerobes $(7,35)$. However, the initial colonization by Proteobacteria in broiler chicken can be of public health risk especially in the context of infection by enteric pathogens such as Salmonella and Campylobacter. An early bloom in these pathogen population in broiler chicken may not be sufficiently countered by the late colonizers, thus resulting in the risk of infection even at market age (15). In this study, a decrease in abundance of Proteobacteria was correlated with an increase in Firmicutes and Actinobacteria population on $18 \mathrm{~d}$. Our findings suggest that early administration of adult feral chicken microbiome could effectively prevent prolonged colonization of facultative anaerobes in chickens.

The microbial profile given in Figure 2 shows the inter-individual variation. The differences between individual birds were more pronounced at $9 \mathrm{~d}$ as indicated by PCA plot (Figure 3 A). Similar variation in microbial composition between the experimental birds has been reported previously $(1,2)$. Similar to our findings where $18 \mathrm{~d}$ old samples clustered together, the microbial communities from older broiler chicken clustered with less variation than 
communities from younger birds (35). Despite the individual variation in the microbial composition, diversity between $9 \mathrm{~d}$ and $18 \mathrm{~d}$ samples was relatively smaller. This is in discordance with previous studies where diversity was lower among the microbial population in the younger birds but increased with age $(14,35)$. The major reason for the increase in the diversity of microbiome with age in these experiments was that the birds were housed in pens or cages and could acquire newer organisms from the habitat. In this experiment, the inoculum served as the sole source of microbes and successful colonization of this microbiome happened by $9 \mathrm{~d}$ of age.

Alternatively, the functional properties of the microbial communities were more stable at $9 \mathrm{~d}$ and $18 \mathrm{~d}$ and were similar to the feral chicken inoculum even while the microbial composition was different. There were no significant differences between the inoculum, $9 \mathrm{~d}$, and $18 \mathrm{~d}$ samples for the functional properties even at level 2 hierarchy using SEED Subsystems database. Similar results for functional properties of chicken cecal microbiome was observed previously $(6,17,35)$. The variability between individuals for the taxonomic profile occurring during the microbial succession did not reflect in the functional profiles in these studies. It has been found that the microbes occupying equivalent niches share similar functional properties even in diverse hosts (41). The microbial assemblage in a previously uninhabited habitat could be driven by equivalence in functional aspects rather than the stochastic nature of microbial colonization. In this study, the host niches being similar, the evenness in functional properties of the communities despite taxonomical variability could only be explained if functionally similar organisms are occupying the equivalent niches.

Chickens act as a reservoir for enteric human pathogens especially, Salmonella. Recently, various serotypes such as Enteritidis, I,[5],12:i:-; Typhimurium; Heidelberg; Hadar; Mbandaka; 
Montevideo; Agona; and Infantis has been associated with Salmonella outbreaks $(42,43)$. These rampant multistate Salmonella outbreaks due to transmission from live poultry reveal the necessity of pursuing studies aimed at control of Salmonella in poultry. The presence of enteric pathogens in poultry was controlled by using antibiotic feed additives (44). Due to the recent FDA regulations to limit the use of antimicrobials in the food supply due to public health concerns, use of such antibiotic feed additives is currently highly controlled. It is pertinent to develop alternatives such as prebiotics and probiotics that could manipulate the microbial community in chicken and thus competitively exclude enteric pathogens. The pioneering work by Nurumi and Rantala in 1973 (10) demonstrated the competitive exclusion of Salmonella by adult chicken microbiome while microbiome from young birds was incapable to prevent the growth of Salmonella (15). The recent outbreaks suggest that this subject should get renewed attention as there are evidence for more Salmonella serotypes adapting to chickens and causing a potential threat to public health (42). Gnotobiotic chickens could serve as excellent models for studying the microbial colonization resistance towards these pathogens and also could be used for development of probiotics as alternatives for controlling the pathogens.

\section{Materials and Methods}

\section{Experiment and sampling}

Feral chickens were obtained locally near Brookings, South Dakota, USA. The feral flock was once a captive flock of mixed breed and has been feral for no less than 8 years. Birds forage on a small grain farm and in surrounding grasslands. Feral chickens were sampled during a routine slaughter for personal meat consumption by the land owners. Gut samples from six birds were collected from the visera following slaughter. The intestine was ligated at distal ileum and distal colon, maintained in ice, and transported immediately to the laboratory and 
stored at $-80 \mathrm{C}$. Protocols used in this study for sample collection were reviewed and approved by the Institutional Animal Care and Use Committee (IACUC) at the South Dakota State University, Brookings, South Dakota. For processing, samples were transferred to a Coy Anaerobic chamber and the contents were expelled into $50 \mathrm{ml}$ sterile conical tubes. Samples were diluted 1:10 (w/v) using anaerobic Brain Heart Infusion broth supplemented with volatile fatty acids and vitamins (BHI-M), mixed by repeated pipetting, and aliquoted into cryovials. Anaerobic DMSO was added at $18 \%$ (final conc) and stored at $-80^{\circ} \mathrm{C}$ until inoculation into young chickens. Simultaneously, an aliquot of the, neat samples from the intestines were streaked on Xylose Lysine Tergitol-4 (XLT-4) agar plates and incubated aerobically at $37^{0} \mathrm{C}$ for $24 \mathrm{~h}$. For preparing inoculant into germ-free chickens, samples were thawed and stock from 6 feral chickens was pooled at equal volume and further diluted 1:10 using anaerobic PBS.

Gnotobiotic chickens were reared using a modified protocol that was described previously (15). Eggs of White Leghorn chickens were acquired from a commercial hatchery, treated with Sporicidin ${ }^{\circledR}$ disinfectant solution (Contec ${ }^{\circledR}$, Inc.), with sterile water and incubated in an incubator, pre-treated with Sporicidin ${ }^{\circledR}$, at $37^{\circ} \mathrm{C}$ at $55 \%$ humidity. Humidity was maintained using a $1 \%(\mathrm{wt} / \mathrm{vol})$ aqueous solution of potassium permanganate. After $19 \mathrm{~d}$ of incubation, eggs were removed from the incubator and candled for viability. Viable eggs were transferred to an UV biosafety cabinet and dipped in Sporicidin ${ }^{\circledR}$ solution for $15 \mathrm{~s}$ then wiped with a sterile cloth saturated with sterile water. Eggs were then transferred to autoclaved egg trays, placed in sterile autoclave bags and transferred immediately to the port of the isolator unit. Eggs were sprayed with 5\% peracetic acid, and after 20 min exposure, were transferred inside the isolators. Eggs were maintained at $37^{\circ} \mathrm{C}$ at $65 \%$ humidity until hatching on $21 \mathrm{~d}$. 
Following hatching, birds were provided ad libitum sterilized water and gammairradiated starter diet (LabDiet@ 5065, Irradiated) designed to meet nutrient requirements of young chickens (Table 1) and monitored daily. On 3 d post-hatch, birds $(n=17)$ were inoculated orally with $300 \mu \mathrm{L}$ of pooled cecal contents. Eight birds were euthanized using cervical dislocation on $9 \mathrm{~d}$ and 9 birds were euthanized on $18 \mathrm{~d}$ post-hatch. The cecal contents were collected for DNA isolation and stored at $-20^{\circ} \mathrm{C}$ until use.

To assess the sterility of the isolator, swabs were collected on $2 \mathrm{~d}$ post-hatch from the egg shells, droppings, and isolator floor and transferred to anaerobic transport media (45) and removed from the isolator. The swabs were then streaked on BHI-M agar plates and incubated aerobically at $37^{0} \mathrm{C}$. The plates were examined for the presence of bacterial colonies after $24 \mathrm{~h}$ and $48 \mathrm{~h}$ of incubation.

\section{Genomic DNA isolation from the cecal contents}

Genomic DNA was isolated using Powersoil DNA isolation kit (Mo Bio Laboratories Inc, CA). Briefly, approximately $100 \mathrm{mg}$ of cecal contents were transferred to bead tubes and samples were homogenized for 2 min using TissueLyser (Qiagen, Germantown, MD). DNA isolation was performed according to manufacturer's protocol and DNA was eluted in $50 \mu \mathrm{L}$ nuclease-free water. The quality of genomic DNA samples was assessed using NanoDrop ${ }^{\mathrm{TM}}$ One (Thermo Fisher Scientific, Wilmington, DE) and quantified using Qubit Fluorometer 3.0 (Invitrogen, Carlsbad, CA). Samples were stored at $-20^{\circ} \mathrm{C}$ until use.

\section{Microbial DNA enrichment and shotgun metagenomics sequencing}

Selective enrichment of bacterial genomic DNA was performed using NEBNext@ Microbiome DNA Enrichment Kit (New England Biolabs, Inc. MA) following methods previously published by our group(29). Briefly, $0.5 \mu \mathrm{g}$ of genomic DNA was treated with $80 \mu 1$ 
of MBD2-Fc bound magnetic beads in the presence of binding buffer and incubated at room temperature for 15 min with rotation. After incubation, beads were separated by keeping the tubes on a magnetic rack for 5 min. The supernatant containing microbial DNA was transferred to a fresh tube. The DNA was further purified using Agencourt AMPure XP beads (Beckman Coulter) and stored at $-20^{0} \mathrm{C}$.

For shotgun metagenome sequencing, the enriched genomic DNA from pooled feral samples, 8 samples from $9 \mathrm{~d}$ and 9 samples from $18 \mathrm{~d}$ post-hatch gnotobiotic chickens was used. The concentrations of genomic DNA samples were adjusted to $0.3 \mathrm{ng} / \mu 1$. Samples were then processed using Nextera XT DNA Sample Prep Kit (Illumina Inc. San Diego, CA) according to manufacturer's protocol. Purified products with unique barcodes were normalized using bead normalization protocol of the manufacturer and equal volumes of normalized libraries were pooled together and diluted in hybridization buffer. The diluted libraries were heat denatured prior to loading to the sequencer. Illumina paired-end sequencing was performed on the Miseq platform using a 2 x 250 paired-end sequencing chemistry.

\section{Sequence data processing}

The raw data files were de-multiplexed and converted to fastq files using Casava v.1.8.2. (Illumina, Inc, San Diego, CA, USA). Fastq files were concatenated and analyzed using MGRAST pipeline (46). The quality control steps in MG-RAST included dereplication, ambiguous base filtering, and length filtering. The taxonomical abundance was analyzed using MG-RAST with Best Hit Classification approach using Refseq database and parameters were limited to minimum e-value of $10^{-5}$, minimum percentage identity of $60 \%$, a minimum abundance of 50 , and a minimum alignment length of 30 amino acids. The functional abundance was analyzed using Hierarchical Classification in MG-RAST using SEED Subsystems database and 
parameters were limited to minimum e-value of $10^{-5}$, minimum percentage identity of $60 \%$, a minimum abundance of 50, and a minimum alignment length of 30 amino acids. The OTU abundance tables were downloaded from MG-RAST and were used for downstream statistical analysis.

\section{Statistical analysis}

The beta diversity between the feral chicken inoculum, $9 \mathrm{~d}$, and $18 \mathrm{~d}$ samples was estimated using Morisita-Horn index in Explicet software (47). The PCA analysis for taxonomical and functional diversity was performed using STAMP (48). Also, the differences in genus-level distribution between $9 \mathrm{~d}$ and $18 \mathrm{~d}$ samples were calculated in STAMP software using White's non-parametric t-test with Storey false discovery rate correction and filtered for a minimum of 200 reads. The differences in phylum-level distribution between $9 \mathrm{~d}$ and $18 \mathrm{~d}$ samples were calculated in GraphPad prism 7.03 (GraphPad Software, San Diego, CA) using non-parametric Mann-Whitney U test. A significant difference was recorded at $\mathrm{P}$ value $<0.05$. Genera-level distribution tables were analyzed using Explicet software. 


\section{Author Contributions}

JS conceived and designed the experiments. MT, SW, SG, and AS performed the experiments. MT analyzed the data. JS, KD, EN, SC, TW and TJ contributed reagents and materials. MT, JS and KD wrote the manuscript. All authors reviewed and approved the manuscript.

\section{Funding}

This work was supported in part by the USDA National Institute of Food and Agriculture, Hatch projects SD00H532-14 and SD00R540-15, and a grant from the South Dakota Governor's Office of Economic Development awarded to JS.

\section{Conflict of Interest Statement}

The authors declare that the research was conducted without any commercial or financial relationships that could be construed as a potential conflict of interest.

\section{References}

1. Stanley D, Denman SE, Hughes RJ, Geier MS, Crowley TM, Chen H, Haring VR, Moore RJ. 2012. Intestinal microbiota associated with differential feed conversion efficiency in chickens. Appl Microbiol Biotechnol 96:1361-9.

2. Park SH, Perrotta A, Hanning I, Diaz-Sanchez S, Pendleton S, Alm E, Ricke SC. 2017. Pasture flock chicken cecal microbiome responses to prebiotics and plum fiber feed amendments. Poult Sci 96:1820-1830.

3. Schokker D, Jansman AJ, Veninga G, de Bruin N, Vastenhouw SA, de Bree FM, Bossers A, Rebel JM, Smits MA. 2017. Perturbation of microbiota in one-day old broiler chickens with antibiotic for 24 hours negatively affects intestinal immune development. BMC Genomics 18:241.

4. Zhenping S, Wenting L, Ruikui Y, Jia L, Honghong L, Wei S, Zhongmie W, Jingpan L, Zhe S, Yuling Q. 2013. Effect of a straw-derived xylooligosaccharide on broiler growth performance, endocrine metabolism, and immune response. Can J Vet Res 77:105-9. 
5. Jorgensen H, Zhao XQ, Knudsen KE, Eggum BO. 1996. The influence of dietary fibre source and level on the development of the gastrointestinal tract, digestibility and energy metabolism in broiler chickens. Br J Nutr 75:379-95.

6. $\quad$ Danzeisen JL, Kim HB, Isaacson RE, Tu ZJ, Johnson TJ. 2011. Modulations of the chicken cecal microbiome and metagenome in response to anticoccidial and growth promoter treatment. PLoS One 6:e27949.

7. Ranjitkar S, Lawley B, Tannock G, Engberg RM. 2016. Bacterial Succession in the Broiler Gastrointestinal Tract. Appl Environ Microbiol 82:2399-410.

8. Barnes EM, Mead GC, Barnum DA, Harry EG. 1972. The intestinal flora of the chicken in the period 2 to 6 weeks of age, with particular reference to the anaerobic bacteria. Br Poult Sci 13:311-26.

9. Jacobs-Reitsma WF, Bolder NM, Mulder RWAW. 1994. Cecal Carriage of Campylobacter and Salmonella in Dutch Broiler Flocks at Slaughter: A One-Year Study. Poultry Science 73:1260-1266.

10. Nurmi E, Rantala M. 1973. New aspects of Salmonella infection in broiler production. Nature 241:210-211.

11. Stern NJ, Eruslanov BV, Pokhilenko VD, Kovalev YN, Volodina LL, Perelygin VV, Mitsevich EV, Mitsevich IP, Borzenkov VN, Levchuk VP, Svetoch OE, Stepanshin YG, Svetoch EA. 2008. Bacteriocins reduce Campylobacter jejuni colonization while bacteria producing bacteriocins are ineffective. Microbial Ecology in Health and Disease 20:74-79.

12. Kim JY, Young JA, Gunther NW, Lee J-L. 2015. Inhibition of Salmonella by Bacteriocin-Producing Lactic Acid Bacteria Derived from U.S. Kimchi and Broiler Chicken. Journal of Food Safety 35:1-12.

13. Oakley BB, Buhr RJ, Ritz CW, Kiepper BH, Berrang ME, Seal BS, Cox NA. 2014. Successional changes in the chicken cecal microbiome during 42 days of growth are independent of organic acid feed additives. BMC Vet Res 10:282.

14. Lu J, Idris U, Harmon B, Hofacre C, Maurer JJ, Lee MD. 2003. Diversity and succession of the intestinal bacterial community of the maturing broiler chicken. Appl Environ Microbiol 69:6816-24.

15. Baba E, Nagaishi S, Fukata T, Arakawa A. 1991. The role of intestinal microflora on the prevention of Salmonella colonization in gnotobiotic chickens. Poult Sci 70:1902-7.

16. Torok VA, Hughes RJ, Mikkelsen LL, Perez-Maldonado R, Balding K, MacAlpine R, Percy NJ, Ophel-Keller K. 2011. Identification and Characterization of Potential Performance-Related Gut Microbiotas in Broiler Chickens across Various Feeding Trials. Applied and Environmental Microbiology 77:5868-5878.

17. Qu A, Brulc JM, Wilson MK, Law BF, Theoret JR, Joens LA, Konkel ME, Angly F, Dinsdale EA, Edwards RA, Nelson KE, White BA. 2008. Comparative metagenomics reveals host specific metavirulomes and horizontal gene transfer elements in the chicken cecum microbiome. PLoS One 3:e2945.

18. Barnes EM. 1979. The intestinal microflora of poultry and game birds during life and after storage. Address of the president of the Society for Applied Bacteriology delivered at a meeting of the society on 10 January 1979. J Appl Bacteriol 46:407-19. 
19. Hanssen I. 1979. A comparison of the microbiological conditions in the small intestine and caeca of wild and captive willow grouse (Lagopus lagopus lagopus). Acta Vet Scand 20:365-71.

20. Schales K, Gerlach H, Kosters J. 1993. Investigations on the aerobic flora and Clostridium perfringens in fecal specimens from free-living and captive capercaillies (Tetrao urogallus L., 1758). Zentralbl Veterinarmed B 40:469-77.

21. Scupham AJ, Patton TG, Bent E, Bayles DO. 2008. Comparison of the cecal microbiota of domestic and wild turkeys. Microb Ecol 56:322-31.

22. Valles Y, Artacho A, Pascual-Garcia A, Ferrus ML, Gosalbes MJ, Abellan JJ, Francino MP. 2014. Microbial succession in the gut: directional trends of taxonomic and functional change in a birth cohort of Spanish infants. PLoS Genet 10:e1004406.

23. Palmer C, Bik EM, DiGiulio DB, Relman DA, Brown PO. 2007. Development of the human infant intestinal microbiota. PLoS Biol 5:e177.

24. Harris ND, Strong DH, Sunde ML. 1968. Intestinal Flora and Chicken Flavor. Journal of Food Science 33:543-457.

25. Gustafsson BE. 1959. Lightweight stainless steel systems for rearing germfree animals. Annals of the New York Academy of Sciences 78:17-28.

26. Szylit O, Charlet G. 1981. Energy and protein retention in holoxenic, axenig and gnotoxenig chickens monoassociated with Lactobacillus SPP. British Poultry Science 22:305-315.

27. Drew MD, Van Kessel AG, Maenz DD. 2003. Absorption of methionine and 2hydroxy-4-methylthiobutoanic acid in conventional and germ-free chickens. Poult Sci 82:1149-53.

28. Hudault S, Bewa H, Bridonneau C, Raibaud P. 1985. Efficiency of various bacterial suspensions derived from cecal floras of conventional chickens in reducing the population level of Salmonella typhimurium in gnotobiotic mice and chicken intestines. Can J Microbiol 31:832-8.

29. Thomas M, Webb M, Ghimire S, Blair A, Olson K, Fenske GJ, Fonder AT, Christopher-Hennings J, Brake D, Scaria J. 2017. Metagenomic characterization of the effect of feed additives on the gut microbiome and antibiotic resistome of feedlot cattle. Sci Rep 7:12257.

30. Johnsson M, Gering E, Willis P, Lopez S, Van Dorp L, Hellenthal G, Henriksen R, Friberg U, Wright D. 2016. Feralisation targets different genomic loci to domestication in the chicken. Nat Commun 7:12950.

31. Gong J, Si W, Forster RJ, Huang R, Yu H, Yin Y, Yang C, Han Y. 2007. 16S rRNA gene-based analysis of mucosa-associated bacterial community and phylogeny in the chicken gastrointestinal tracts: from crops to ceca. FEMS Microbiol Ecol 59:147-57.

32. Sergeant MJ, Constantinidou C, Cogan TA, Bedford MR, Penn CW, Pallen MJ. 2014. Extensive microbial and functional diversity within the chicken cecal microbiome. PLoS One 9:e91941.

33. Zhu XY, Zhong T, Pandya Y, Joerger RD. 2002. 16S rRNA-based analysis of microbiota from the cecum of broiler chickens. Appl Environ Microbiol 68:124-37.

34. Wei S, Morrison M, Yu Z. 2013. Bacterial census of poultry intestinal microbiome. Poult Sci 92:671-83. 
35. Awad WA, Mann E, Dzieciol M, Hess C, Schmitz-Esser S, Wagner M, Hess M. 2016. Age-Related Differences in the Luminal and Mucosa-Associated Gut Microbiome of Broiler Chickens and Shifts Associated with Campylobacter jejuni Infection. Front Cell Infect Microbiol 6:154.

36. Pan D, Yu Z. 2014. Intestinal microbiome of poultry and its interaction with host and diet. Gut Microbes 5:108-19.

37. David LA, Maurice CF, Carmody RN, Gootenberg DB, Button JE, Wolfe BE, Ling AV, Devlin AS, Varma Y, Fischbach MA, Biddinger SB, Dutton RJ, Turnbaugh PJ. 2014. Diet rapidly and reproducibly alters the human gut microbiome. Nature 505:559-63.

38. Jost T, Lacroix C, Braegger CP, Chassard C. 2012. New insights in gut microbiota establishment in healthy breast fed neonates. PLoS One 7:e44595.

39. Jakobsson HE, Abrahamsson TR, Jenmalm MC, Harris K, Quince C, Jernberg C, Bjorksten B, Engstrand L, Andersson AF. 2014. Decreased gut microbiota diversity, delayed Bacteroidetes colonisation and reduced Th1 responses in infants delivered by caesarean section. Gut 63:559-66.

40. Koenig JE, Spor A, Scalfone N, Fricker AD, Stombaugh J, Knight R, Angenent LT, Ley RE. 2011. Succession of microbial consortia in the developing infant gut microbiome. Proc Natl Acad Sci U S A 108 Suppl 1:4578-85.

41. Fan L, Reynolds D, Liu M, Stark M, Kjelleberg S, Webster NS, Thomas T. 2012. Functional equivalence and evolutionary convergence in complex communities of microbial sponge symbionts. Proc Natl Acad Sci U S A 109:E1878-87.

42. CDC. August 21, 2017 2017. Reports of Selected Salmonella Outbreak Investigations. https://www.cdc.gov/salmonella/outbreaks.html. Accessed 9/29/2017.

43. Jackson BR, Griffin PM, Cole D, Walsh KA, Chai SJ. 2013. Outbreak-associated Salmonella enterica Serotypes and Food Commodities, United States, 19982008. Emerging Infectious Diseases 19:1239-1244.

44. Goodnough MC, Johnson EA. 1991. Control of Salmonella enteritidis infections in poultry by polymyxin B and trimethoprim. Appl Environ Microbiol 57:785-8.

45. Alfa M, Lee A. 1982. A transport method for swab specimens submitted for aerobic and anaerobic bacteriology. J Clin Pathol 35:555-60.

46. Meyer F, Paarmann D, D'Souza M, Olson R, Glass EM, Kubal M, Paczian T, Rodriguez A, Stevens R, Wilke A, Wilkening J, Edwards RA. 2008. The metagenomics RAST server - a public resource for the automatic phylogenetic and functional analysis of metagenomes. BMC Bioinformatics 9:386.

47. Robertson CE, Harris JK, Wagner BD, Granger D, Browne K, Tatem B, Feazel LM, Park K, Pace NR, Frank DN. 2013. Explicet: graphical user interface software for metadata-driven management, analysis and visualization of microbiome data. Bioinformatics 29:3100-1.

48. Parks DH, Tyson GW, Hugenholtz P, Beiko RG. 2014. STAMP: statistical analysis of taxonomic and functional profiles. Bioinformatics 30:3123-4. 


\section{Tables and Figures}
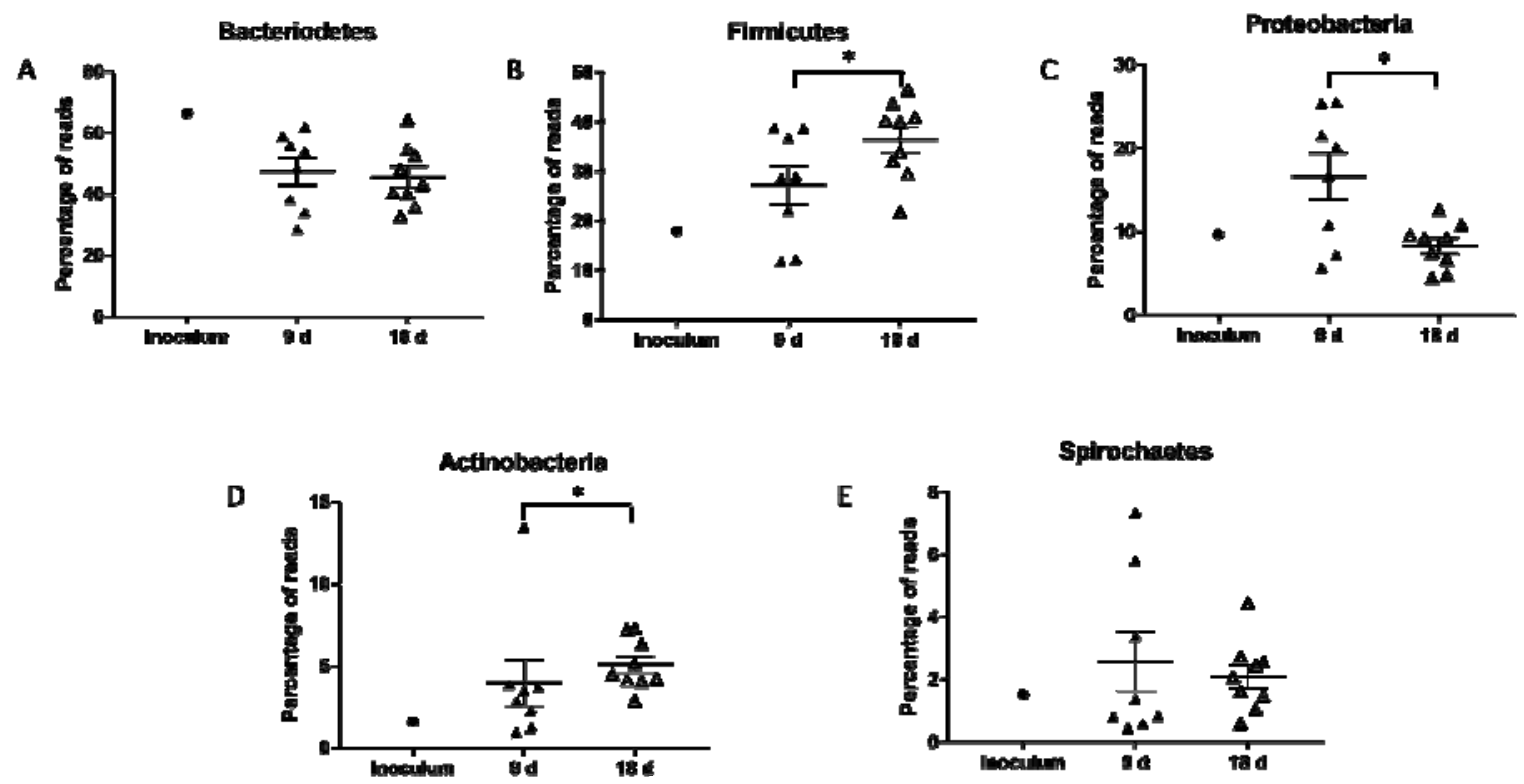

Figure 1. Taxonomical distribution of the major phyla in the inoculum and gnotobiotic chicken gut at $9 \mathrm{~d}$ and $18 \mathrm{~d}$. Inoculum was derived from 6 healthy feral chicken. Germ-free chicks were inoculated on $3 \mathrm{~d}$ post-hatch and euthanized on $9 \mathrm{~d}(\mathrm{n}=8)$ and $18 \mathrm{~d}(\mathrm{n}=9)$ post-hatch. Cecal contents were collected for DNA isolation. DNA was sequenced using the Miseq 2x250 pairedend sequencing platform. Phylogenetic tables were generated in MG-RAST. The statistical difference in phyla distribution between $9 \mathrm{~d}$ and $18 \mathrm{~d}$ samples was calculated using nonparametric Mann-Whitney U test. A) Bacteroidetes B) Firmicutes C) Proteobacteria D) Actinobacteria and E) Spirochetes. * represents $\mathrm{P}>0.05$. 


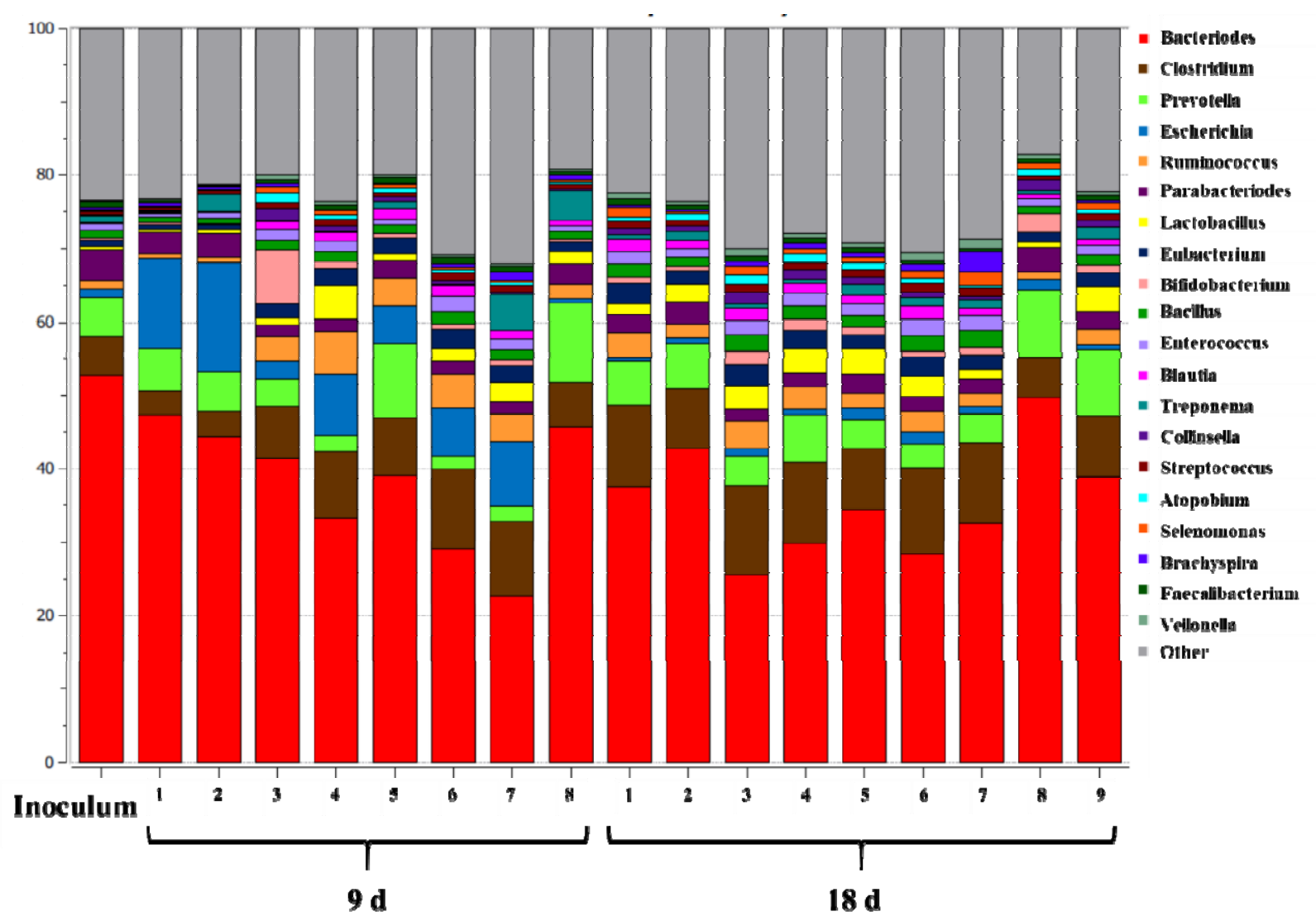

Figure 2. Genera-level distribution of gut microbiome in the gnotobiotic chicken inoculated with intestinal material from feral chickens. The pooled inoculum, derived from 6 healthy feral chicken was orally inoculated to gnotobiotic chicken on $3 \mathrm{~d}$ after hatch. Birds were euthanized on $9 d(n=8)$ and $18 d(n=9)$ of age and cecal contents were collected for DNA isolation. The metagenomic functional analysis was performed in MG-RAST using Refseq database with maximum e-value at $10^{-5}$ value, minimum percentage identity at $60 \%$, minimum alignment length of 30 amino acids and abundance 50. Phylogenetic tables were generated in MG-RAST and analysis was conducted using Explicet software. 

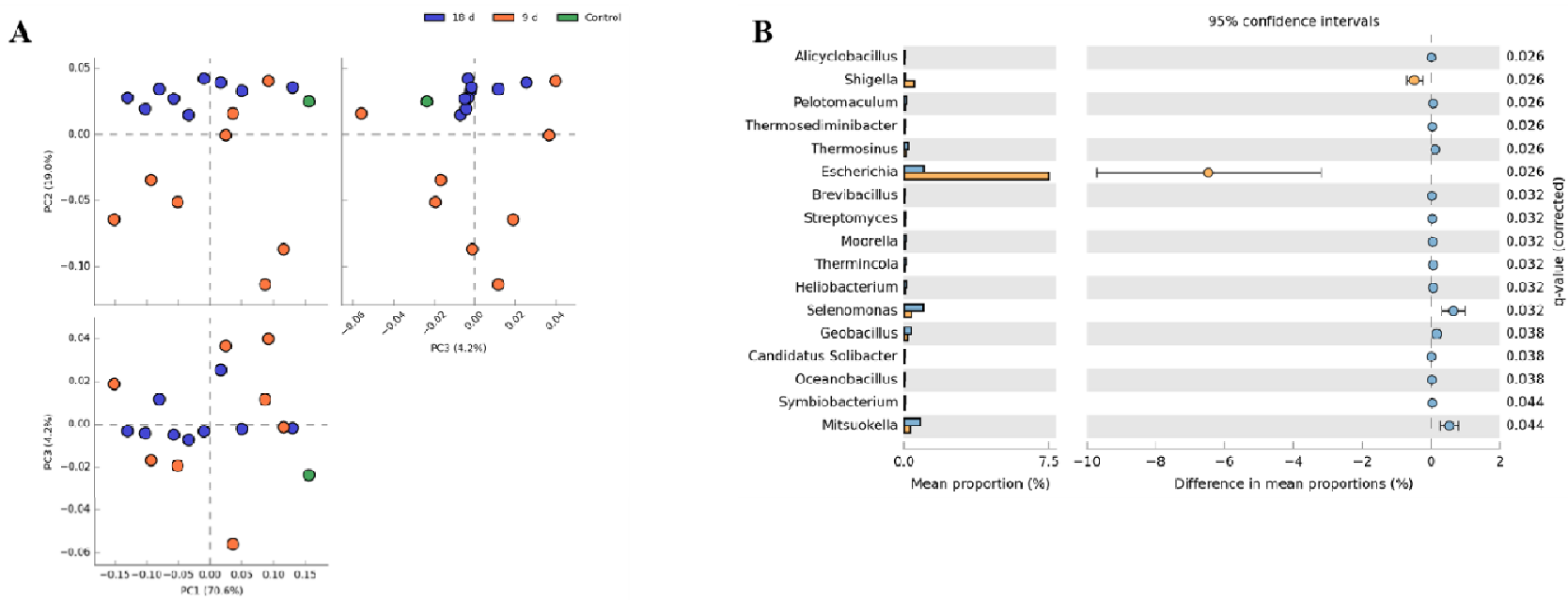

Figure 3. Principle coordinate analysis (PCA) of taxonomical diversity in gnotobiotic chicken.

Donor material derived from 6 healthy feral chicken was orally inoculated to gnotobiotic chicken

on $3 \mathrm{~d}$ after hatch. A) PCA analysis showed that the $18 \mathrm{~d}$ samples from inoculated gnotobiotic chicken was distributed closer to the inoculum when compared to $9 \mathrm{~d}$ samples. B) The major differences at genus level distribution were the reduced abundance of Escherichia and Shigella in the $18 \mathrm{~d}$ samples compared to the $9 \mathrm{~d}$ samples while Selenomonas, Geobacillus, and Mitsuokella increased. 

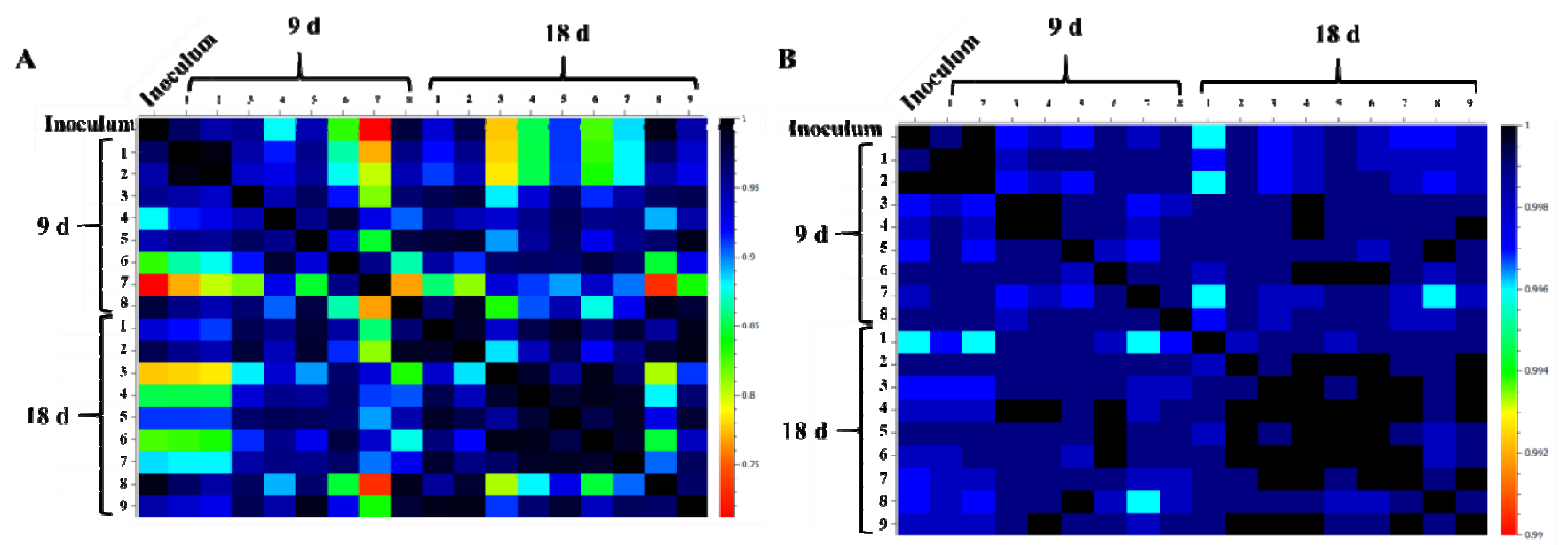

Figure 4. Comparison of taxonomical and functional $\beta$ diversities between feral chicken-derived inoculum and gnotobiotic chicken gut samples on $9 \mathrm{~d}$ and $18 \mathrm{~d}$. The $\beta$ diversities were measured using Morisita-Horn similarity index in Explicet software. The indices range between 0 to 1 where 1 is considered similar and 0 considered dissimilar. Taxonomically, individual variations were observed between the inoculum and gnotobiotic chicken samples while the functional characteristics of the gnotobiotic chicken communities were closely similar to the inoculum. 


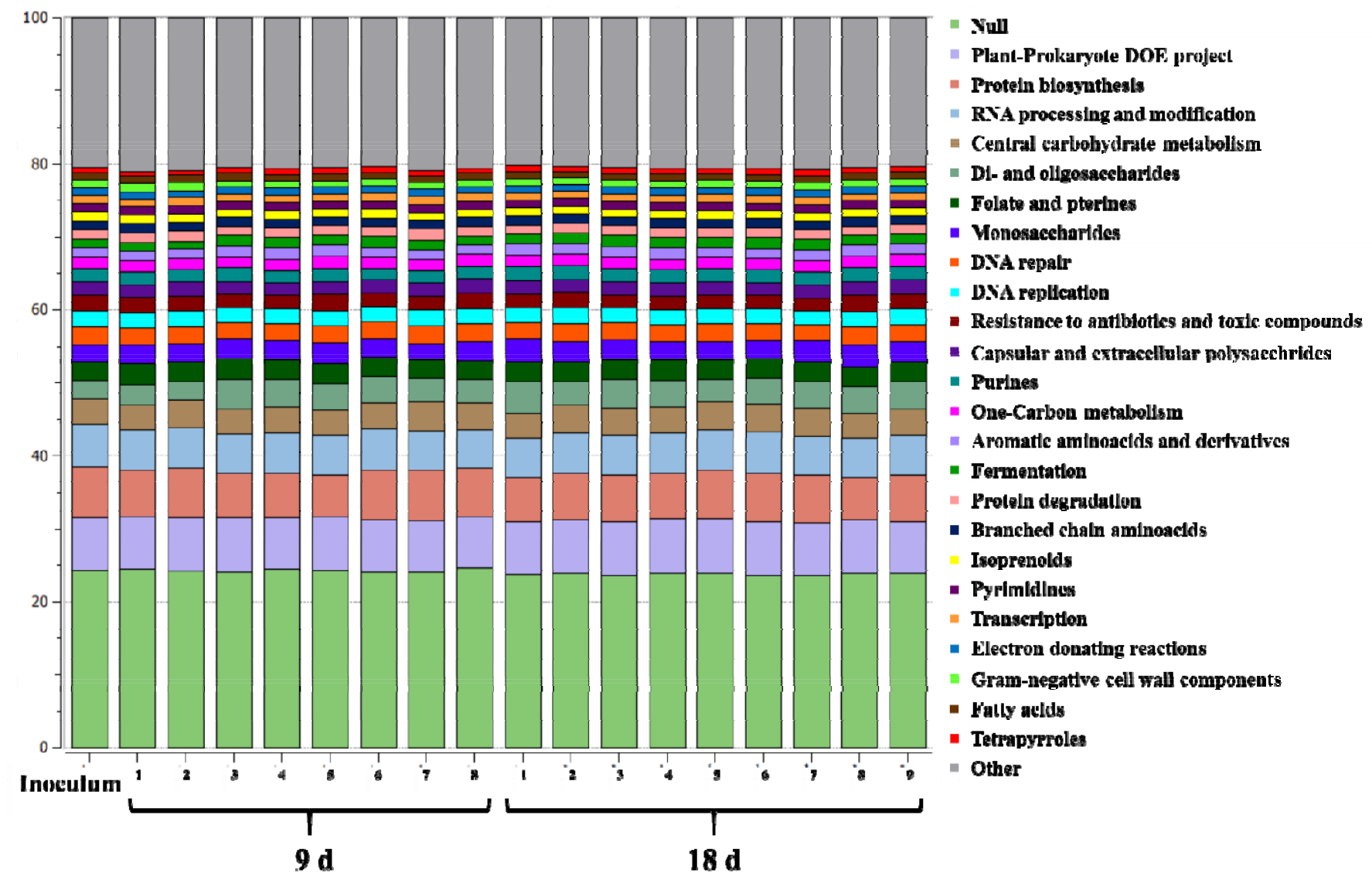

Figure 5. Predicted functional profile of the microbiome in feral and gnotobiotic chicken. The pooled inoculum was derived from 6 healthy feral chicken. Birds were inoculated on $3 \mathrm{~d}$ after hatch, were euthanized on $9 d(n=8)$ and $18 d(n=9)$ of age and cecal contents were collected for DNA isolation. The metagenomic functional analysis was performed in MG-RAST using Subsystems database with maximum e-value at $10^{-5}$ value, minimum percentage identity at $60 \%$, minimum alignment length of 30 amino acids and abundance 50. 
Table 1. Nutritional composition and energy content of the LabDiet ${ }^{\circledR} 5065$, Irradiated diet

Composition in $\%$

Protein

Fat (ether extract)

Fat (acid hydrolysis)

Fiber (max)

Nitrogen-Free Extract

Minerals

Energy source

Protein

Fat (ether extract)

Carbohydrates

Total Energy (kcal/g)

22.1

2.8

55.6

5.3

25.4

10.7

63.9

3.48 